\title{
Barycentric rational interpolation with asymptotically monitored poles
}

\author{
Richard Baltensperger
}

Received: 8 October 2009 / Accepted: 22 July 2010 /

Published online: 20 August 2010

(C) Springer Science+Business Media, LLC 2010

\begin{abstract}
We present a method for asymptotically monitoring poles to a rational interpolant written in barycentric form. Theoretical and numerical results are given to show the potential of the proposed interpolant.
\end{abstract}

Keywords Interpolation • Rational interpolation • Exponential convergence • Monitoring poles

Mathematics Subject Classifications (2010) Primary 65D05 • 41A05 • $41 \mathrm{~A} 20 \cdot 41 \mathrm{~A} 25$

\section{Introduction}

In 1986, Schneider and Werner presented in [1] a new algorithm for rational interpolation based on the barycentric formula and showed that this form offers various advantages in comparison with other representations. Among them were criteria for the location of poles of the interpolant and the ease of differentiating the rational function. Extensions and applications of the work of Schneider and Werner have been made in the following decades, see for example (in chronological order) [2,3] or [4] (for a survey).

In the present work we shall be interested in the problem of interpolating a function $f$ in an interval $I=[a, b]$ by a global interpolant defined on the same interval. We shall review some of the known results concerning barycentric

\footnotetext{
R. Baltensperger $(\bowtie)$

College of Engineering and Architecture of Fribourg,

University of Applied Sciences of Western Switzerland,

Bd de Pérolles 80, 1705 Fribourg, Switzerland

e-mail: richard.baltensperger@hefr.ch
} 
rational (and polynomial) interpolation (Section 2). In Section 3, we shall present the method of attaching poles to the polynomial and of shifting the points without loosing the convergence property. In the following section, we shall present a way of aymptotically monitoring poles to a rational interpolant. This method generalizes existing work. Finally we shall demonstrate the quality of this new interpolant (with aymptotically monitored and optimized poles) on numerical examples.

\section{Barycentric rational interpolation}

Let $x_{0}, x_{1}, \ldots, x_{N}$ be $N+1$ distinct points of an interval $I=[a, b]$ of $\mathbb{R}$ and $f_{j}:=f\left(x_{j}\right), j=0(1) N$, be the corresponding values of a function $f$ defined on the real line. Let $\mathcal{P}_{m}$ be the space of all polynomials of degree at most $m$ and $\mathcal{R}_{m, n}$ be the set of all rational functions with numerator in $\mathcal{P}_{m}$ and denominator in $\mathcal{P}_{n}$. Any rational function $r_{N}[f]$ in $\mathcal{R}_{N, N}$ for which $r_{N}[f]\left(x_{j}\right)=f_{j}$, $j=0(1) N$, can be written as

$$
r_{N}[f](x)=\frac{\sum_{j=0}^{N} \frac{u_{j}}{x-x_{j}} f_{j}}{\sum_{j=0}^{N} \frac{u_{j}}{x-x_{j}}},
$$

a formula known as the barycentric form of the rational interpolant $r_{N}[f]$. The derivation is fairly simple: the denominator $q$ of $r_{N}[f]$ may be written in its Lagrangian form (see [5]) as

$$
q(x)=L(x) \sum_{j=0}^{N} \frac{w_{j}}{x-x_{j}} q_{j}, \quad q_{j}:=q\left(x_{j}\right),
$$

where $w_{j}:=1 / \prod_{i \neq j}\left(x_{j}-x_{i}\right)$ (the so-called weights) and $L(x):=\prod_{i=0}^{N}\left(x-x_{i}\right)$. Since $r_{N}[f]\left(x_{j}\right)=f_{j}$ and $q\left(x_{j}\right)=q_{j}$, one has $p\left(x_{j}\right)=p_{j}=f_{j} q_{j}$ for the numerator $p$ of $r_{N}[f]$. Writing $p$ in its Lagrangian form

$$
p(x)=L(x) \sum_{j=0}^{N} \frac{w_{j}}{x-x_{j}} p_{j}
$$

and letting $u_{j}:=w_{j} q_{j}$, one has (1).

Conversely, as presented in [3] or [4], one can easily construct a function $r_{N}[f] \in \mathcal{R}_{N, N}$ with the interpolating property: let $u_{0}, u_{1}, \ldots, u_{N}$ be $N+1$ real numbers, different from 0 ; the rational function (1) interpolates $f_{j}$ between the $x_{j}$. 
2.1 From rational to polynomial interpolation

Note that the unique polynomial $P_{N}[f] \in \mathcal{P}_{N}$ for which $P_{N}[f]\left(x_{j}\right)=f_{j}$, $j=0(1) N$, is obtained by imposing $q(x) \equiv 1$ in (2), so that the $u_{j}$ 's in (1) are replaced by the $w_{j}$ 's:

$$
P_{N}[f](x)=\frac{\sum_{j=0}^{N} \frac{w_{j}}{x-x_{j}} f_{j}}{\sum_{j=0}^{N} \frac{w_{j}}{x-x_{j}}} .
$$

This formula is the barycentric form of the interpolating polynomial and is known to be a very stable way of evaluating the interpolating polynomial, see [5] or [6] for more details.

For "special" points, such as equidistant points or Chebyshev points of the first and the second kind (see for example [7,8] or [5]), formulas for the weights $w_{j}$ are known. Moreover, as the weights now arise in the denominator as well as in the numerator, any common factor that does not explicitly depend on the nodes may be simplified, leading to simplified weights $w_{j}^{s}$. For example, for the Chebyshev points of the second kind $x_{j}=\cos \frac{j \pi}{N}, j=0(1) N$, one has

$$
w_{j}^{s}=(-1)^{j} \delta_{j}, \quad \delta_{j}= \begin{cases}1 / 2, & j=0, N \\ 1, & j \neq 0, N\end{cases}
$$

Formula (4) for Chebyshev points of the second kind is extremely stable and, if $f$ is analytic inside and on an ellipse $E_{\rho}$ with foci at \pm 1 and the sum of the ellipse's major and minor axes equals $2 \rho, \rho>1$, one has exponential convergence of $P_{N}[f]$ toward $f$ :

$$
\left|P_{N}[f](x)-f(x)\right|=\mathcal{O}\left(\rho^{-N}\right) .
$$

This very nice property has been used in several applications, see $[9,10]$ or [11] for instance.

However, due to the clustering of the points near the extremities, the information (that is the $f_{j}$ 's) is badly distributed over the interval and could lead to mediocre approximation of functions with shocks close to the center. Moreover, there is an ill-conditioning of the derivatives of $P_{N}[f]$ near the extremities, see [10] for instance.

\section{Back to rational interpolation}

Knowing that the set $\mathcal{R}_{N, N}$ is larger than (and contains) the space $\mathcal{P}_{N}$, one can hope to find interpolants in $\mathcal{R}_{N, N}$ (that is, finding points $x_{j}$ and/or weights $u_{j}$ 
in (1)) that lead to better convergence properties of the interpolant $r_{N}[f]$ as compared to $P_{N}[f]$. This question has already been addressed and answered (in part) and we shall review some of known results in the following two subsections.

\subsection{Attaching poles}

Sometimes the interpolated function $f$ has poles whose location is known or may be guessed a priori. Let us assume that we know $K$ poles $z_{i}, i=1(1) K$, with respective multiplicities $v_{i}\left(v:=\sum_{i=1}^{K} v_{i} \leq N\right)$ of $f$.

We can therefore rewrite the function $f$ as a quotient of two functions $h$ and $d$ where $d(x):=a \prod_{i=1}^{K}\left(x-z_{i}\right)^{\nu_{i}}, a \neq 0 \in \mathbb{C}$ arbitrary, and construct a rational interpolant with preassigned poles. We obtain a rational function $r_{N}^{*}[f]$ that interpolates $f$ between the $x_{j}$ and has $K$ preassigned poles $z_{i}$ (assuming that all the $z_{i}$ are different from the interpolation points $x_{j}$ and that $P_{N}[h]\left(z_{i}\right)=$ $\left.h\left(z_{i}\right) \neq 0\right)$

$$
r_{N}^{*}[f](x)=\frac{P_{N}[h](x)}{d(x)} .
$$

Let $d_{j}:=d\left(x_{j}\right)$ be the values of the denominator $d$ at the interpolation points. Since $\operatorname{deg}(d)=v \leq N$, we may rewrite $d$ as

$$
d(x)=P_{N}[d](x)=L(x) \sum_{j=0}^{N} \frac{w_{j}}{x-x_{j}} d_{j} .
$$

We have $h_{j}:=h\left(x_{j}\right)=f_{j} d_{j}$ and therefore, the numerator $h$ can be approximated by the interpolating polynomial of degree $\leq N$

$$
P_{N}[h](x)=\sum_{j=0}^{N} h_{j} L_{j}(x)=L(x) \sum_{j=0}^{N} \frac{w_{j} d_{j}}{x-x_{j}} f_{j} .
$$

Dividing (8) by (7), we find

$$
r_{N}^{*}[f](x)=\frac{P_{N}[h](x)}{P_{N}[d](x)}=\frac{\sum_{j=0}^{N} \frac{b_{j}}{x-x_{j}} f_{j}}{\sum_{j=0}^{N} \frac{b_{j}}{x-x_{j}}}
$$

with $b_{j}:=w_{j} d_{j}, j=0(1) N . r_{N}^{*}[f]$ interpolates $f$ and has $K$ attached poles $z_{1}, \ldots, z_{K}$. The degree of its numerator is $\leq N$ and that of its denominator is $=v$. Therefore, $r_{N}^{*}[f] \in \mathcal{R}_{N, v}$ and one may hope that $r_{N}^{*}[f]$ approximates $f$ better than $P_{N}[f]$ based on the same interpolation points (if the location of the poles is known or guessed a priori). This construction has been presented in [12] and successfully applied for example in [13] and [14]. 


\subsection{Changing the points}

Instead of changing the weights $w_{j}$, one may move the points. A first attempt was made in [2], where the author suggested to keep the same Chebyshev weights (5) independently of the interpolation points. What is the convergence property of the resulting interpolant? Unfortunately the exponential convergence (6) is lost (even if $f$ is analytic inside and on an ellipse $E_{\rho}$ with foci at \pm 1 and the sum of the ellipse's major and minor axes equal to $2 \rho, \rho>1$ ). Recently Floater and Hormann [15] showed what Berrut suspected and was conjectured in [16]: an $\mathcal{O}\left(h^{2}\right)$ convergence for that interpolant, at least for equidistant points.

How can we "move" the points (in view, for example, of better capturing the fronts of a function) whithout loosing the exponential convergence property? A solution has been proposed in [16]. One has to take Berrut's rational interpolant of [2], (that is taking (4) with the weights defined by (5)) and move the points $x_{k}$ conformally from the Chebyshev position. This yields

$$
r_{N}[f](x)=\frac{\sum_{j=0}^{N} \frac{w_{j}^{s}}{x-x_{j}} f_{j}}{\sum_{j=0}^{N} \frac{w_{j}^{s}}{x-x_{j}}}, \quad x_{j}=g(\cos (j \pi / N)),
$$

with $g$ a "good" function to be defined.

The following theorem proven in [16] gives the settings in which the rational interpolant can be found.

Theorem 1 Let $\mathcal{D}_{1}, \mathcal{D}_{2}$ be two domains of $\mathbb{C}$ containing $J=[-1,1]$, respectively $I(\in \mathbb{R})$, let $g$ be a conformal map $\mathcal{D}_{1} \rightarrow \mathcal{D}_{2}$ such that $g(J)=I$, and $f$ be a function $\mathcal{D}_{2} \rightarrow \mathbb{C}$ such that the composition $f \circ g: \mathcal{D}_{1} \rightarrow \mathbb{C}$ is analytic inside and on an ellipse $C_{\rho}\left(\subset \mathcal{D}_{1}\right), \rho>1$, with foci at \pm 1 and with the sum of its major and minor axes equal to $2 \rho$. Let $r_{N}[f]$ be the rational function given by (10). Then for every $x \in[-1,1]$,

$$
\left|r_{N}[f](x)-f(x)\right|=\mathcal{O}\left(\rho^{-N}\right) .
$$

The only remaining problem is to find a "good" conformal map g. Several maps have been proposed (and used) in the past, among them the Kosloff and Tal-Ezer map [17], which moves the points towards the interior of the domain. This map has proven to be interesting for solving partial differential equations [18] or boundary value problems [19]. One further has the Bayliss and Turkel map [20] and its generalization [21], which move the points towards front(s) of the interpolated function $f$ (see [21] and [22] for an application to boundary value problems) and, more recently, the Tee and Trefethen map [23] and the 
Hale and Tee map [24], which also shift the points towards the front, but in a more elegant manner. These last maps were used to solve partial differential equations with fronts.

\section{Changing the points and the weights}

We have seen in Subsection 3.1 how to attach poles to an interpolating polynomial (that is, how to change the weights of the interpolant) and in Subsection 3.2 how to move the points without loosing convergence. A fairly natural question arise: Can we asympotically attach or asymptotically monitor poles to a rational interpolant written in barycentric form?

Again we will assume that the location of $K$ poles of the interpolated function is known a priori so that $f=h / d$. We shall now repeat the steps from (7) to (9) albeit in the rational case. One may approximate the numerator $h$ and the denominator $d$ of $f$ by two rational interpolants $r_{N}[h]$ (resp. $r_{N}[d]$ ) with the same denominator and write these in barycentric form (1):

$$
r_{N}[h](x)=\frac{\sum_{j=0}^{N} \frac{u_{j}}{x-x_{j}} h_{j}}{\sum_{j=0}^{N} \frac{u_{j}}{x-x_{j}}}, \quad r_{N}[d](x)=\frac{\sum_{j=0}^{N} \frac{u_{j}}{x-x_{j}} d_{j}}{\sum_{j=0}^{N} \frac{u_{j}}{x-x_{j}}} .
$$

Dividing $r_{N}[h]$ by $r_{N}[d]$ and using that $h_{j}=f_{j} d_{j}, \quad j=0(1) N$, we get

$$
R_{N}^{*}[f](x)=\frac{r_{N}[h](x)}{r_{N}[d](x)}=\frac{\sum_{j=0}^{N} \frac{b_{j}}{x-x_{j}} f_{j}}{\sum_{j=0}^{N} \frac{b_{j}}{x-x_{j}}} \in \mathcal{R}_{N, N},
$$

where the $b_{j}$ 's are given by $b_{j}:=u_{j} d_{j} j=0(1) N$ (with the $u_{j}$ 's different from 0). $R_{N}^{*}[f]$ interpolates $f$ (that is $R_{N}^{*}[f]\left(x_{j}\right)=f_{j}$ ) and has almost the $K$ attached poles $z_{1}, \ldots, z_{K}$. This is because $r_{N}[d]$ is an approximation of $d$ and therefore $r_{N}[d]\left(z_{i}\right) \approx d\left(z_{i}\right)=0(i=1(1) K)$ if $r_{N}[d]$ is a "good" approximant of the denominator $d$ (see also [25], Remark 2.1). Further poles may arise in $\mathbb{C}$ but will move infinitely far as $N \rightarrow \infty$ (again if $r_{N}[d]$ is a "good" approximant of $d$ ).

\subsection{Convergence}

In view of keeping the exponential convergence of the rational interpolant $R_{N}^{*}[f]$ towards $f$ (if $f$ is analytic inside a domain containing $[-1,1]$ ), it is 
natural to use the weights defined by $b_{j}=w_{j}^{s} d_{j}, j=0(1) N$, and use the transformed Chebyshev points. In that case, the rational interpolant reads

$$
R_{N}^{*}[f](x)=\frac{\sum_{j=0}^{N} \frac{b_{j}}{x-x_{j}} f_{j}}{\sum_{j=0}^{N} \frac{b_{j}}{x-x_{j}}}, \quad x_{j}:=g(\cos (j \pi / N)),
$$

with $g$ a conformal map. The following theorem gives us the settings in which the rational interpolant can be found.

Theorem 2 Let the same hypotheses as in Theorem 1 hold. Assume further that the location of $K$ poles of the function $f \circ g$ is known a priori so that $(f \circ g)=$ $(h \circ g) /(d \circ g)$. Let $R_{N}^{*}[f]$ be the rational function given by (13). Then for every $x \in[-1,1]$,

$$
\left|R_{N}^{*}[f](x)-f(x)\right|=\mathcal{O}\left(\rho^{-N}\right)
$$

Proof $f \circ g$ is analytic inside and on an ellipse $C_{\rho}$ with foci at \pm 1 and its axes equal to $2 \rho, \rho>1$. On the other hand, $d \circ g$ is analytic inside and on an ellipse $C_{\tilde{\rho}}$ with foci at \pm 1 and its axes equal to $2 \tilde{\rho}, \tilde{\rho} \geq \rho$ and we know from Theorem 1 that $\left|r_{N}[d](x)-d(x)\right|=\mathcal{O}\left(\tilde{\rho}^{-N}\right)$. Since $f \circ g=(h \circ g) /(d \circ g), h \circ g$ is analytic inside and on the ellipse $C_{\rho}$ and $\left|r_{N}[h](x)-h(x)\right|=\mathcal{O}\left(\rho^{-N}\right)$ (again by Theorem 1).

Therefore we get (by applying the same computation as in the proof of Theorem 4 in [16])

$$
\begin{aligned}
R_{N}^{*}[f](x)=\frac{r_{N}[h](x)}{r_{N}[d](x)} & =\frac{h(x)+\mathcal{O}\left(\rho^{-N}\right)}{d(x)+\mathcal{O}\left(\tilde{\rho}^{-N}\right)} \\
& =\frac{h(x) / d(x)+\mathcal{O}\left(\rho^{-N}\right)}{1+\mathcal{O}\left(\tilde{\rho}^{-N}\right)} \\
& =f(x)+\mathcal{O}\left(\rho^{-N}\right) .
\end{aligned}
$$

\section{Another successful strategy}

In [21] and [22], the authors have successfully applied a method proposed by Kosloff and Tal-Ezer [17]. It consists in replacing the Chebyshev points by their images under a conformal map (which preserves spectral convergence). They consider the physical space $x$ as the image $g(y)$ of another coordinate space $y$. In this space $y$, one can approximate a function $F(y)\left(=f\left(g^{-1}(x)\right)\right)$ 
by the interpolating polynomial $\left(P_{N}[F](y)\right)$ or by the interpolating rational interpolant $\left(r_{N}^{*}[F](y)\right)$ with attached poles $v_{i}=g^{-1}\left(z_{i}\right)$. Transplanted back to the (physical) $x$-space, this results in the two following interpolants (written in barycentric form), which are note rational any longer. The first reads

$$
P_{N}[F](y)=\frac{\sum_{j=0}^{N} \frac{w_{j}^{s}}{y-y_{j}} F_{j}}{\sum_{j=0}^{N} \frac{w_{j}^{s}}{y-y_{j}}}=\frac{\sum_{j=0}^{N} \frac{w_{j}^{s}}{g^{-1}(x)-g^{-1}\left(x_{j}\right)} f_{j}}{\sum_{j=0}^{N} \frac{w_{j}^{s}}{g^{-1}(x)-g^{-1}\left(x_{j}\right)}}=: A_{N}[f](x),
$$

where the $y_{j}=\cos (j \pi / N), j=0(1) N$ are the Chebyshev points of the second kind and the $w_{j}^{s}$ are the corresponding polynomial weights (5). The second interpolant (with attached poles) is given by

$$
r_{N}^{*}[F](y)=\frac{\sum_{j=0}^{N} \frac{c_{j}}{y-y_{j}} F_{j}}{\sum_{j=0}^{N} \frac{c_{j}}{y-y_{j}}}=\frac{\sum_{j=0}^{N} \frac{c_{j}}{g^{-1}(x)-g^{-1}\left(x_{j}\right)} f_{j}}{\sum_{j=0}^{N} \frac{c_{j}}{g^{-1}(x)-g^{-1}\left(x_{j}\right)}}=: A_{N}^{*}[f](x),
$$

where $c_{j}, j=0(1) N$ are given by $c_{j}:=w_{j}^{s} \cdot \prod_{i=1}^{K}\left(y_{j}-v_{i}\right)$.

Remark Although the interpolants (15) and (16) are close to their correponding rational interpolants (10) and (13), they are not the same. For example, when using the former for solving differential equations (as was done in [22]), one has to apply the chain rule for differentiating them. For the rational interpolants, one can simply use the formulae proposed (for example) in [18], which leads to less complicated formulae and computer programs.

\section{Numerical evidence}

In all of the following examples we compared the numerical results obtained with the following six interpolants:

1. The "classical" interpolating polynomial (written in barycentric form) based on Chebyshev points (4), denoted $P_{N}[f]$ in the following tables and figures.

2. The "transplanted" interpolating polynomial $A_{N}[f]$ given by (15).

3. The rational interpolant (10) based on transformed Chebyshev points (1) with simplified Chebyshev weights $w_{j}^{s}$ from (5), denoted $r_{N}[f]$ in the following. 
4. The interpolating rational function based on Chebyshev points with attached poles $r_{N}^{*}[f](9)$.

5. The "transplanted" interpolating polynomial with attached poles $A_{N}^{*}[f](16)$.

6. The rational interpolant based on transformed Chebyshev points (with simplified Chebyshev weights $w_{j}^{s}$ from (5)) with asymptotically monitored poles $R_{N}^{*}[f](13)$.

We interpolated three different functions. The $\infty$-error was computed using $\|h-k\|_{\infty} \approx \max _{i}\left|h\left(\tilde{x}_{i}\right)-k\left(\tilde{x}_{i}\right)\right|$ where $\tilde{x}_{i}=-1+\frac{2 i}{1000}, i=0(1) 1000$. All computations were conducted on a Windows based machine using MATLAB 7.4.

\subsection{Example 1}

In our test with the first example we compared all six interpolants. We chose the function

$$
f(x)=\frac{\sin (m x)}{1+a x^{2}}, \quad \text { with } a=100 \text { and } m=10 .
$$

For this function (the solution of the test problem proposed in [14]), the two poles are $z_{1,2}= \pm i \sqrt{1 / a}$. We have interpolated $f$ using $N=10$ Chebyshevpoints of the second kind $\left(y_{j}=\cos \frac{j \pi}{N}\right)$. As conformal map $g$ we took the Kosloff Tal-Ezer map [17]

$$
g(y)=\frac{\arcsin (\alpha y)}{\arcsin (\alpha)}
$$

with a fixed $\alpha$ set to 0.9 .

In Figs. 1 and 2, we give the various interpolants (dashed line) and compare them with the original function (solid line). We see visually that the rational interpolant with preassigned poles $R_{N}^{*}[f]$ (13) gives the best results, as confirmed by Table 1, which also shows that monitoring two poles improves the approximation by a factor of 10 .

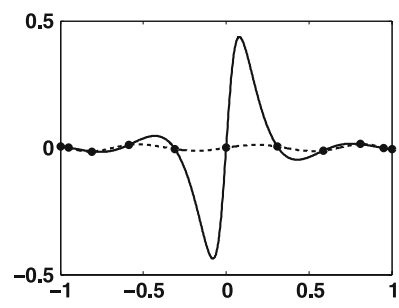

(a)

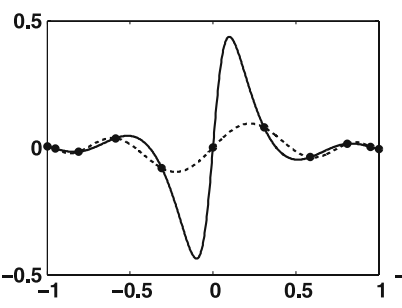

(b)

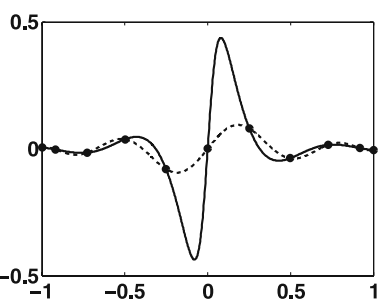

(c)

Fig. 1 (a) $P_{N}[f]$ and $f$; (b) $A_{N}[f]$ and $f$; (c) $r_{N}[f]$ and $f$ 


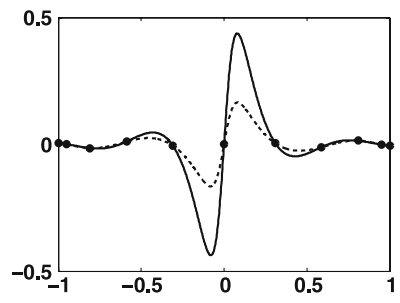

(d)

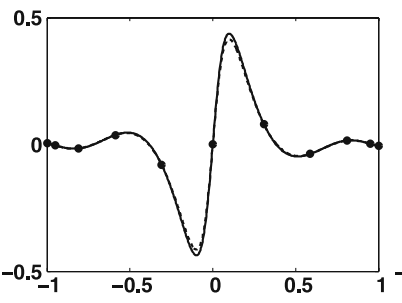

(e)

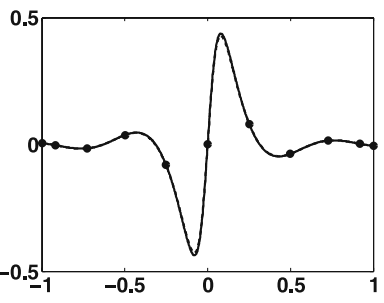

(f)

Fig. 2 (d) $r_{N}^{*}[f]$ and $f$; (e) $A_{N}^{*}[f]$ and $f$; (f) $R_{N}^{*}[f]$ and $f$

\subsection{Example 2}

In the second example we have interpolated the function (displayed in Fig. 3) $f(x)=\sin (3 x) e^{-x^{2}}-e^{-x^{2} / \epsilon}$ with $\epsilon$ set to $\epsilon=10^{-2}$. We chose $N=40$ Chebyshev points and used again the Kosloff and Tal-Ezer map (17) with $\alpha$ set to 0.9.

In that case, as we did not know exactly the location of the poles, we followed the method used by Berrut and Mittelman in [13] for the interpolants $r_{N}^{*}[f], A_{N}^{*}[f]$ and $R_{N}^{*}[f]$. We optimized two (monitored for $R_{N}^{*}[f]$ ) poles via the "fminsearch" function of MATLAB. The results obtained in this case are reported in Table 2.

We can see there that attaching (monitoring for $R_{N}^{*}[f]$ ) two (optimized) poles to the interpolating functions leads to a gain of one power of ten in the approximation of the function, a result already obtained with Example 1.

In Fig. 4 we give the approximation error (in semilog- $y$ chart) of the various interpolants with increasing $N$. We can see the exponential convergence of the various interpolants $(f$ is analytic in $[-1,1])$. The solid line represents the approximation error of $P_{N}[f]$, the dashed line that of $r_{N}^{*}[f]$, the dotted lines the approximation errors with $A_{N}[f]$ and $r_{N}[f]$ (mingled dotted lines on the chart as the approximation errors of both interpolants are almost the same) and the dotted dashed lines the approximation error of $A_{N}^{*}[f]$ and $R_{N}^{*}[f]$ (again mingled as the errors are about the same).

\subsection{Example 3}

In the third example (taken from [21]) we have interpolated the function $f(x)=u_{1}(x)+u_{2}(x)$ with $u_{1}(x)=e^{1 /(x+1.2)}+\cos \pi(x+0.5), u_{2}(x)=\frac{\operatorname{erf}(\delta(x+0.5))}{\operatorname{erf}(\delta)}$

Table $1 \infty$-error of the six interpolants in Example 1

\footnotetext{
${ }^{(1)}$ In the space $y$ defined in Section 5

${ }^{(2)}$ Two monitored poles
}

\begin{tabular}{lll}
\hline Approximant & Poles & Error \\
\hline$P_{N}[f]$ & - & $4.3 \cdot 10^{-1}$ \\
$A_{N}[f]$ & - & $3.8 \cdot 10^{-1}$ \\
$r_{N}[f]$ & - & $3.8 \cdot 10^{-1}$ \\
$r_{N}^{*}[f]$ & $\pm i \sqrt{1 / a}$ & $2.7 \cdot 10^{-1}$ \\
$A_{N}^{*}[f]$ & same $^{(1)}$ & $2.3 \cdot 10^{-2}$ \\
$R_{N}^{*}[f]$ & same $^{(2)}$ & $1.0 \cdot 10^{-2}$ \\
\hline
\end{tabular}


Fig. 3 The function $f$ of Example 2

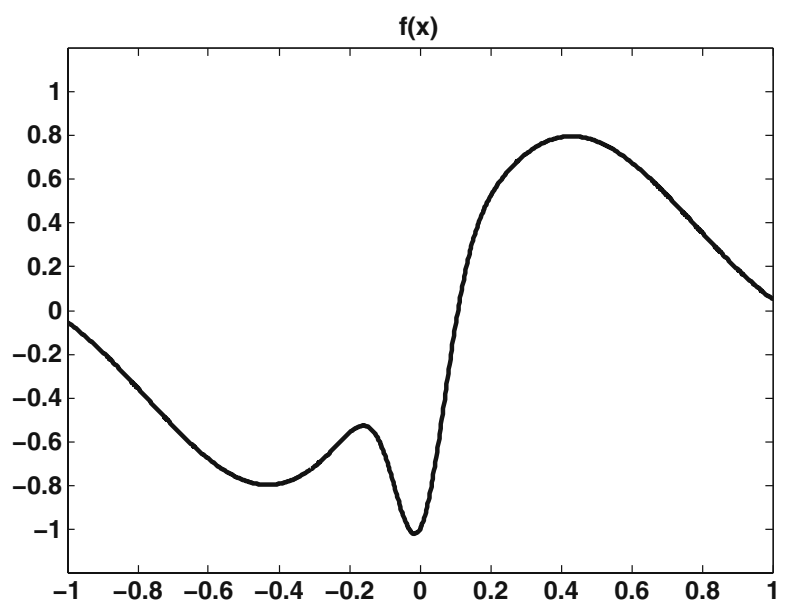

and $\delta=10^{2} \sqrt{0.5}$. $f$ is plotted in Fig. 5, where one can see the function on the interval $[-1,1]$ and a zoom of it.

We chose $N=100$ Chebyshev points and used the Bayliss and Turkel map (one shock) [20] given by

$$
\begin{aligned}
g(y) & =\frac{1}{\alpha} \tan (\lambda(y-\mu))+\beta, \quad \lambda=\frac{\gamma+\delta}{2}, \quad \mu=\frac{\gamma-\delta}{\gamma+\delta}, \\
\gamma & =\arctan (\alpha(1+\beta)), \quad \delta=\arctan (\alpha(1-\beta)) .
\end{aligned}
$$

In that case, two (monitored for $R_{N}^{*}[f]$ ) poles or/and the parameters $\alpha$ and $\beta$ have been optimized via "fminsearch" in MATLAB.

We observe in Table 3 that optimizing the parameters $\alpha$ and $\beta$ lead to a gain of eight powers of ten in the approximation of $f$ (see also Fig. 6)! Monitoring and optimizing two poles for $R_{N}^{*}[f]$ leads to a gain of two more powers of ten in the approximation of $f$, in line with the results obtained in [22].

In Fig. 6 we give the approximation error (in semilog- $y$ chart) of the various interpolants with increasing $N$. The analyticity of $f$ leads to the exponential convergence of the various interpolants. As in Fig. 4, the solid line represents the approximation error of $P_{N}[f]$, the dashed line the approximation error of $r_{N}^{*}[f]$, the dotted lines the approximation error of $A_{N}[f]$ and $r_{N}[f]$ and the dotted dashed lines the approximation error of $A_{N}^{*}[f]$ and $R_{N}^{*}[f]$. We see that

Table $2 \infty$-error of the six interpolants in Example 2

(3) Two optimized and monitored poles

\begin{tabular}{lll}
\hline Approximant & Poles & Error \\
\hline$P_{N}[f]$ & - & $4.84 \cdot 10^{-3}$ \\
$A_{N}[f]$ & - & $4.25 \cdot 10^{-4}$ \\
$r_{N}[f]$ & - & $4.52 \cdot 10^{-4}$ \\
$r_{N}^{*}[f]$ & $-1.07 \cdot 10^{-7} \pm 2.15 \cdot 10^{-1} i$ & $1.75 \cdot 10^{-4}$ \\
$A_{N}^{*}[f]$ & $2.09 \cdot 10^{-7} \pm 2.63 \cdot 10^{-1} i$ & $1.29 \cdot 10^{-5}$ \\
$R_{N}^{*}[f]$ & $1.06 \cdot 10^{-6} \pm 2.63 \cdot 10^{-1} i^{(3)}$ & $1.30 \cdot 10^{-5}$ \\
\hline
\end{tabular}


Fig. 4 The function $f$ of Example 2 interpolated with increasing number of points $(N=10,20,40,80,160,320)$

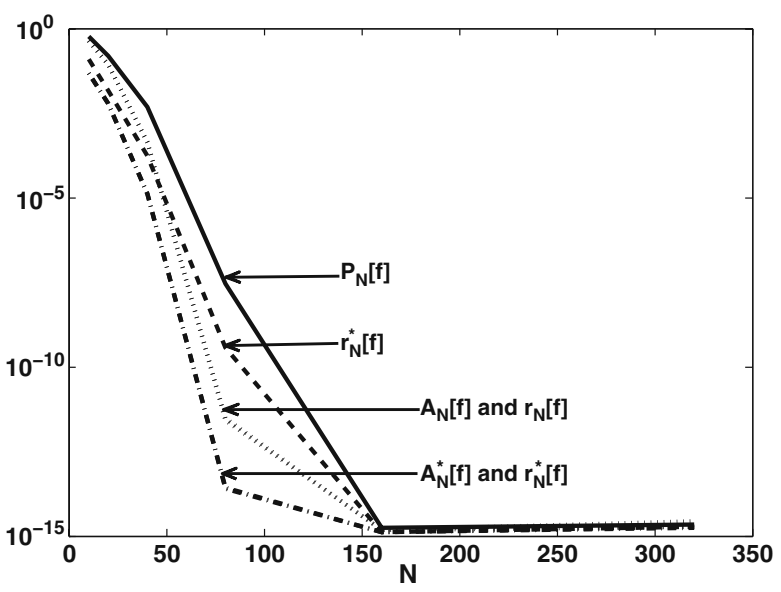

the convergence behaviour of $A_{N}[f]$ and $r_{N}[f]$ (resp. $A_{N}^{*}[f]$ and $R_{N}^{*}[f]$ ) are similar.

\subsection{Comparison of maps}

Several maps have been presented in recent articles, see for example [23, 26] or [24]. If one knows the (approximate) location (for example) of two conjugate poles $\delta \pm i \varepsilon$ of a function $f$, one may use the Hale and Tee map defined by the composition $g=h_{2} \circ h_{1}$ of two functions given by

$$
\begin{aligned}
& h_{1}(z)=\sqrt[4]{m} \operatorname{sn}\left(\frac{2 K}{\pi} \arcsin (z) \mid m\right), \\
& h_{2}(z)=A-C\left(\frac{1-\cos (\theta)}{2(z-1)}+\frac{1+\cos (\theta)}{2(z+1)}\right) .
\end{aligned}
$$
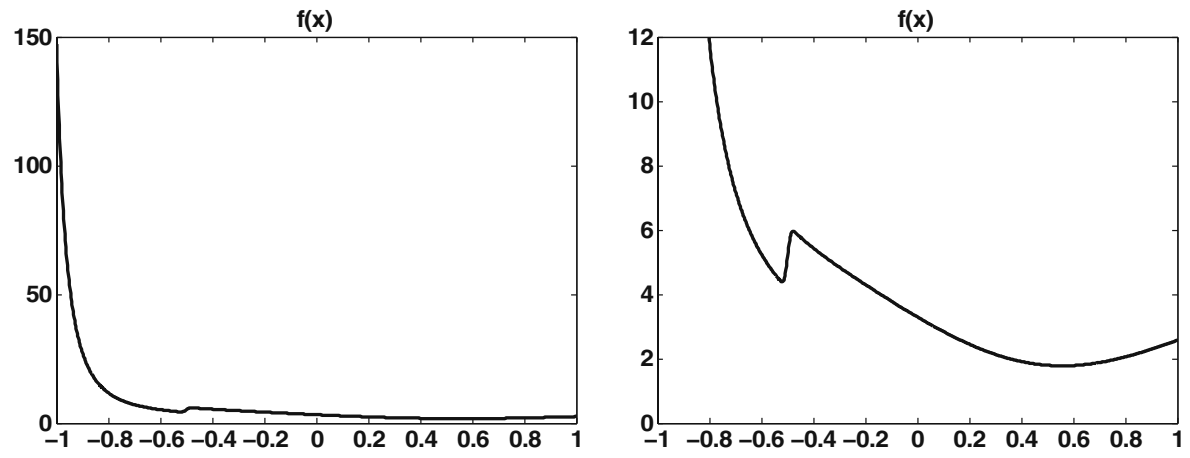

Fig. 5 Example 3 (left) and zoom of Example 3 (right) 
Table $3 \infty$-error of the six interpolants in Example 3

\begin{tabular}{llll}
\hline Approximant & Poles & $\alpha, \beta$ & Error \\
\hline$P_{N}[f]$ & - & & $1.69 \cdot 10^{-1}$ \\
$A_{N}[f]$ & - & $\alpha=7.41, \beta=-0.52$ & $9.38 \cdot 10^{-9}$ \\
$r_{N}[f]$ & - & $\alpha=7.61, \beta=-0.52$ & $9.35 \cdot 10^{-9}$ \\
$r_{N}^{*}[f]$ & $-4.99 \cdot 10^{-1} \pm 2.00 \cdot 10^{-2} i$ & - & $1.69 \cdot 10^{-1}$ \\
$A_{N}^{*}[f]$ & $-1.09 \cdot 10^{0} \pm 3.83 \cdot 10^{-3} i$ & $\alpha=8.55, \beta=-0.51$ & $3.82 \cdot 10^{-11}$ \\
$R_{N}^{*}[f]$ & $-1.13 \cdot 10^{0} \pm 3.64 \cdot 10^{-3} i^{(4)}$ & $\alpha=8.32, \beta=-0.51$ & $1.35 \cdot 10^{-11}$ \\
\hline
\end{tabular}

(4) Optimized and monitored poles

Fig. 6 The function $f$ of Example 3 interpolated with increasing number of points $(N=10,20,40,80,160,320)$

Fig. 7 The function $f$ of Example 3 interpolated with increasing number of points $(N=10,20,40,80,160,320)$
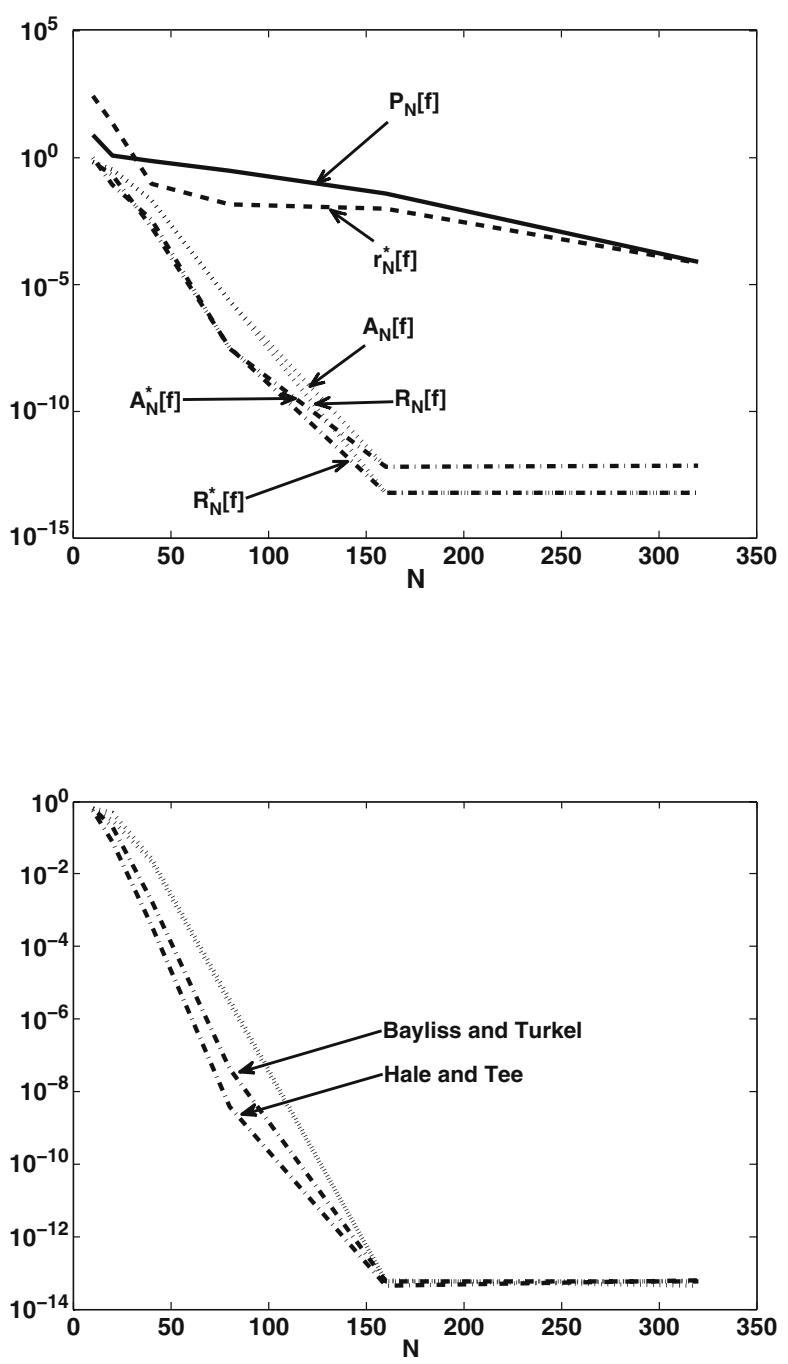
$K(m)$ is the complete elliptic integral of the first kind, $\operatorname{sn}(\cdot \mid m)$ the Jacobi elliptic sine function [27] and the parameters $A, C, \theta$ and $m$ are determined by the four real conditions $h_{2}\left( \pm m^{1 / 4}\right)= \pm 1, \operatorname{Re}\left(h_{2}\left(z_{1}\right)\right)=\delta$ and $\operatorname{Im}\left(h_{2}\left(z_{1}\right)\right)=\varepsilon$, see [26] or [24] for more details and the related functions for more than two conjugate poles.

In Fig. 7 we give the approximation error (in semilog- $y$ chart) of $r_{N}[f]$ and $R_{N}^{*}[f]$ for various $N$. We compare the results obtained with the Bayliss and Turkel map (18) and the Hale and Tee map (19). In both cases we optimized two parameters ( $\alpha$ and $\beta$ for the Bayliss and Turkel map resp. $\delta$ and $\varepsilon$ for the Hale and Tee map) for $r_{N}[f]$ and two parameters ( $\alpha$ and $\beta$ resp. $\delta$ and $\varepsilon$ ) and two asymptotically monitored poles for $R_{N}^{*}[f]$. The interpolated function $f$ is again the function defined in Subsection 6.3.

We can see the exponential convergence of the various interpolants $(f$ is analytic in $[-1,1])$. As in Fig. 4, the dotted lines represent the approximation error of $r_{N}[f]$ (mingled dotted lines on the chart as the approximation error is almost the same with both maps) and the dotted dashed lines represent the approximation error of $R_{N}^{*}[f]$. We see that using the Hale and Tee map enhance the convergence behaviour of $R_{N}^{*}[f]$ by approximatively a factor of 10 (for moderate values of $N$ ).

\section{Conclusion}

In the present article, we have presented a way to asymptotically monitor poles to an interpolating rational function written in barycentric form. This approximant could represent an interesting alternative to existing methods for solving differential equations by means of pseudospectral methods.

Acknowledgements Part of this work was done while the author was at the Department of Mathematics of the University of Fribourg, Switzerland. The author would like to thank especially Jean-Paul Berrut and Jean-Pierre Gabriel of that Department for fruitful and stimulating discussions on all sorts of subjects. The author would like to thank the unknown referees whose comments have considerably improved the text of the present paper.

\section{References}

1. Schneider, C., Werner, W.: Some new aspects of rational interpolation. Math. Comput. 47, 285-299 (1986)

2. Berrut, J.-P.: Rational functions for guaranteed and experimentally well-conditioned global interpolation. Comput. Math. Appl. 15, 1-16 (1988)

3. Berrut, J.-P., Mittelmann, H.D.: Lebesgue constant minimizing linear rational interpolation of continuous functions over the interval. Comput. Math. Appl. 33, 77-86 (1997)

4. Berrut, J.-P., Baltensperger, R., Mittelmann, H.D.: Recent developments in barycentric rational interpolation. Trends and applications in constructive approximation. Internat. Ser. Numer. Math. (ISNM) 151, 27-51 (2005)

5. Berrut, J.-P., Trefethen, L.N.: Barycentric lagrange interpolation. SIAM Rev. 46, 501-517 (2004)

6. Higham, N.: The numerical stability of barycentric Lagrange interpolation. IMA J. Numer. Anal. 24, 547-556 (2004) 
7. Salzer, H.E.: Lagrangian interpolation at the Chebyshev points $x_{n, v}=\cos (v \pi / n)$; some unnoted advantages. Comput. J. 15, 156-159 (1972)

8. Henrici, P.: Essentials of Numerical Analysis with Pocket Calculator Demonstrations. Wiley, New York (1982)

9. Battles, Z., Trefethen, L.N.: An extension of MATLAB to continuous functions and operators. SIAM J. Sci. Comput. 25, 1743-1770 (2004)

10. Trefethen, L.N.: Spectral Methods in MATLAB. SIAM, Philadelphia (2001)

11. Fornberg, B.: A Practical Guide to Pseudospectral Methods. Cambridge University Press, Cambridge (1996)

12. Berrut, J.-P.: The barycentric weights of rational interpolation with prescribed poles. J. Comput. Appl. Math. 86, 45-52 (1997)

13. Berrut, J.-P., Mittelmann, H.D.: Rational interpolation through the optimal attachment of poles to the interpolating polynomial. Numer. Algorithms 23, 315-328 (2000)

14. Baltensperger, R., Berrut, J.-P., Dubey, Y.: The linear rational pseudospectral method with preassigned poles. Numer. Algorithms 33, 53-63 (2003)

15. Floater, M.S., Hormann, K.: Barycentric rational interpolation with no poles and high rates of approximation. Numer. Math. 107, 315-331 (2007)

16. Baltensperger, R., Berrut, J.-P., Noël, B.: Exponential convergence of a linear rational interpolant between transformed Chebyshev points. Math. Comput. 68, 1109-1120 (1999)

17. Kosloff, D., Tal-Ezer, H.: A modified Chebyshev pseudospectral method with an $\mathcal{O}\left(N^{-1}\right)$ time step restriction. J. Comput. Phys. 104, 457-469 (1993)

18. Baltensperger, R., Berrut, J.-P.: The linear rational collocation method. J. Comput. Appl. Math. 134, 243-258 (2001)

19. Berrut, J.-P., Baltensperger, R.: The linear rational pseudospectral method for boundary value problems. BIT 41, 868-879 (2001)

20. Bayliss, A., Turkel, E.: Mappings and accuracy for Chebyshev pseudo-spectral approximations. J. Comput. Phys. 101, 349-359 (1992)

21. Berrut, J.-P., Mittelmann, H.D.: Adaptive point shifts in rational approximation with optimized denominator. J. Comput. Appl. Math. 164-165, 81-92 (2004)

22. Berrut, J.-P., Mittelmann, H.D.: Optimized point shifts and poles in the linear rational pseudospectral method for boundary value problems. J. Comput. Phys. 204, 292-301 (2005)

23. Tee, T.W., Trefethen, L.N.: A rational spectral collocation method with adaptively transformed Chebyshev grid points. SIAM J. Sci. Comput. 28, 1798-1811 (2006)

24. Hale, N., Tee, T.W.: Conformal maps to multiply-slit domains and applications. SIAM J. Sci. Comput. 31, 3195-3215 (2009)

25. Berrut, J.-P., Mittelmann, H.D.: Point shift in rational interpolation with optimized denominator. In: Algorithms for Approximation IV: Proceedings of the 2001 International Symposium, pp. 420-427 (2002)

26. Tee, T.W.: An adaptive rational spectral method for differential equations with rapidly varying solutions. Ph.D. Thesis, University of Oxford (2006)

27. Abramowitz, M., Stegun, I.A.: Handbook of Mathematical Functions with Formulas, Graphs and Mathematical Tables. Dover, New York (1964) 